\title{
Aplicação da nanotecnologia na engenharia de alimentos: uma revisão
}

\author{
Application of nanotechnology in food engineering: a review \\ Aplicación de la nanotecnología en ingeniería alimentaria: una revisión
}

Recebido: 05/01/2022 | Revisado: 10/01/2022 | Aceito: 15/01/2022 | Publicado: 17/01/2022

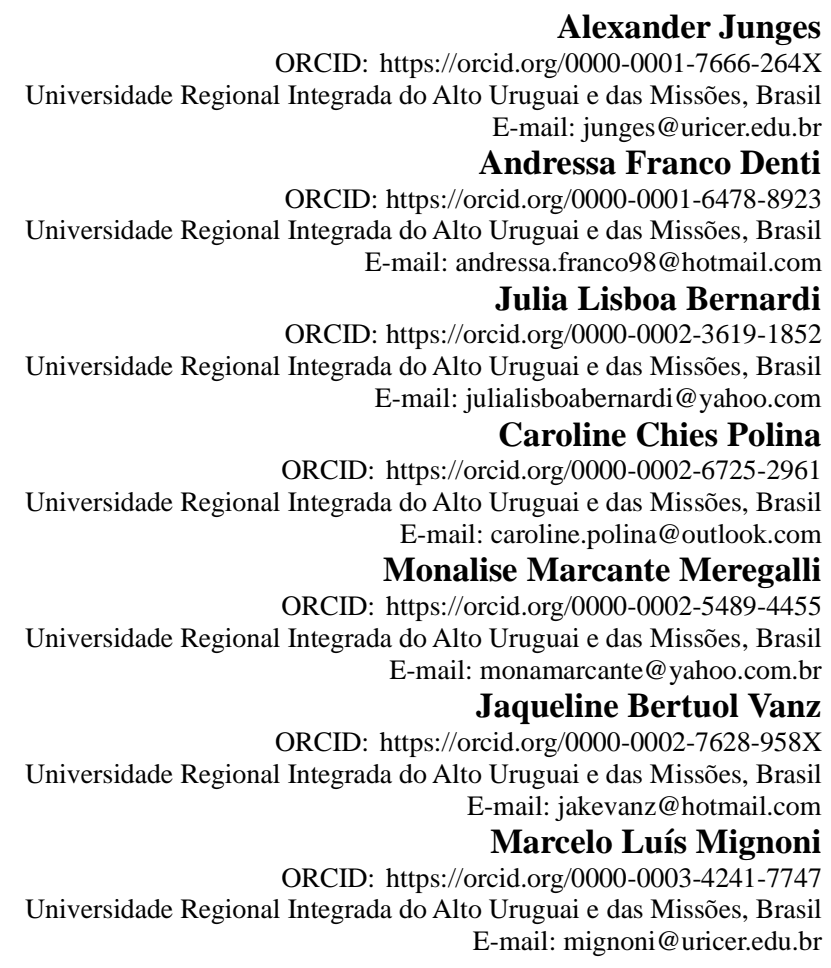

\section{Resumo}

A nanotecnologia pode ser definida como uma expressão genérica de um complexo de tecnologias, técnicas e processos voltados ao desenvolvimento de novos materiais em escala nanométrica e podendo ser aplicada em diferentes setores industriais, inclusive no alimentício. Tendo em vista o aumento do consumo de produtos biodegradáveis e aspectos ambientais do mesmo, diversas tecnologias vêm sendo empregadas na indústria alimentícia, buscando evitar a produção exagerada de resíduos e o desperdício, otimizando a cadeia produtiva em diferentes pontos onde possa reduzir o custo de fabricação, aumentar a vida de prateleira ou adicionar algum ingrediente funcional. Este estudo apresenta uma revisão de uma série de aplicações da nanotecnologia na produção de alimentos, em especial nos produtos cárneos, lácteos, doces, frutas e vegetais, embalagens e nanosensores.

Palavras-chave: Nanotecnologia; Tecnologia industrial; Produtos alimentícios.

\begin{abstract}
Nanotechnology can be defined as a generic expression of a complex of technologies, techniques and processes aimed at the development of new materials on a nanometric scale and can be applied in different industrial sectors, including foods. In view of the increased consumption of biodegradable products and its environmental aspects, several technologies have been used in the food industry, seeking to avoid the exaggerated production and waste, optimizing the production chain at different points where it can reduce the manufacturing cost, increase shelf life or add some functional ingredient. This study presents a review of a series of applications of nanotechnology in food production, especially in meat, dairy, sweets, fruits and vegetables, packaging and nanosensors.
\end{abstract}

Keywords: Nanotechnology; Industrial technology; Food products.

\section{Resumen}

La nanotecnología puede definirse como una expresión genérica de un conjunto de tecnologías, técnicas y procesos orienta-dos al desarrollo de nuevos materiales a escala nanométrica y pueden aplicarse en diferentes sectores industriales, incluido el alimentario. Ante el mayor consumo de productos biodegradables y sus aspectos ambientales, se han utilizado diversas tecnologías en la industria alimentaria, buscando evitar la producción exagerada y residuos, 
optimizando la cadena de producción en diferentes puntos donde se puede reducir el costo de fabricación, aumente la vida útil o agregue algún ingrediente funcional. Este estudio presenta una revisión de una serie de aplicaciones de la nanotecnología en la producción de alimentos, especialmente en carnes, lácteos, dulces, frutas y verduras, envases y nanosensores.

Palabras clave: Nanotecnología; Tecnología industrial; Productos alimenticios.

\section{Introdução}

A nanociência é uma área com grande potencial de aplicação tecnológica, sendo a nanotecnologia considerada uma valiosa ferramenta para o aperfeiçoamento e desenvolvimento de novos produtos (Azeredo, 2012; Mihindukulasuriya \& Kim, 2014; Martins et al., 2015).

A nanotecnologia acarreta melhorias na produção e técnicas de processamento de alimentos, aumentando a vida de prateleira de alimentos, mudanças de texturas, modificação de sabor, qualidade e segurança de produtos alimentícios (Greiner et al., 2009; Martins et al., 2015). Pode ser definida como uma expressão genérica de um complexo de tecnologias, técnicas e processos voltados ao desenvolvimento de novos materiais em escala nanométrica, a partir da manipulação de moléculas ou átomos (Ferreira et al., 2018), podendo ser aplicada em diferentes setores industriais.

$\mathrm{Na}$ indústria de alimentos, observa-se a importância e a influência da nanotecnologia em todas as etapas, desde produção agrícola até o armazenamento e posterior consumo. A cada dia é desenvolvido um novo produto com embalagens ativas e inteligentes com maiores resistências térmicas, mecânicas e biodegradáveis (Handford et al., 2014; Martins et al., 2015).

Os sistemas de embalagens nanotecnológicas estão se tornando cada vez mais sofisticados, graças à rápida evolução da ciência. $\mathrm{O}$ crescente desenvolvimento das tecnologias está levando a inovação no campo de embalagens inteligentes e utilizadas na conservação e armazenamento de alimentos.

Os antimicrobianos presentes em embalagens de frutas e verduras podem estender a vida útil e adiar a deterioração dos alimentos. Este processo se faz necessário para reduzir quantidade de produtos químicos necessários nos alimentos. As embalagens não biodegradáveis têm inúmeras desvantagens e configuram ameaças ao meio ambiente devido a quantidade de agentes tóxicos. A utilização de nanomateriais como aditivos em alimentos também tem sido objetivo de estudos para ganho de sabor, cor, estabilidade no processamento e aumento da vida de prateleira (Handford et al., 2014; Martins et al., 2015).

Nanopartículas de $\mathrm{SiO}_{2}, \mathrm{TiO}_{2}$ e $\mathrm{Ag}$ são empregadas, respectivamente, como agente antiaglomerante, agente de revestimento e agente antimicrobiano. As nanopartículas de $\mathrm{TiO}_{2}$ são empregadas em chocolates, iogurtes, queijos entre outros grupos alimentícios. (Greiner, 2009; Handford et al., 2014; Martins et al., 2015).

Moura et al (2012), realizaram um experimento com filmes nanocompósitos de prata de vários tamanhos com hidroxipropilmetilcelulose. Neste estudo pode-se contatar que os de menor tamanho, apresentavam maior proteção contra vapores de água, Escherichia coli e Staphylococcus aureus. Este estudo sinalizou a ênfase ao desenvolvimento de outros trabalhos com uso de polímeros biodegradáveis como por exemplo a celulose.

Nesta revisão bibliográfica, abordou-se um levantamento sobre conceitos e utilização da nanotecnologia em alimentos, especialmente em produtos cárneos, lácteos, doces, frutas, vegetais, nanossensores e embalagens.

\section{Metodologia}

Para a elaboração do estudo a metodologia empregada fundamentou-se em uma revisão narrativa da literatura realizada por meio da análise de artigos científicos, monografias, dissertações, teses e livros através das seguintes bases de dados e sites de busca: Springer, ACS Publications, Science Direct, Scielo, Google Scholar e Wiley Online Library, no período de 2011 a 2021, sem desconsiderar publicações importantes de anos anteriores. 


\section{Nanotecnologia em Alimentos}

O termo nanotecnologia é derivado do prefixo grego "nannos", que significa anão, e de "techne" e "logos", correspondendo a ofício e conhecimento, respectivamente (Fernandes, 2019). É a utilização de partículas nanométricas bilionésima parte do metro - na escala de $1 \mathrm{a} 100 \mathrm{~nm}$ (Barros, 2011), que apresentam comportamento diferenciado quando comparadas à macropartículas da mesma substância, principalmente pela maior superfície de contato nas nanoestruturas (Aguiar et al., 2021; Martins et al., 2015).

Há diferentes tipos de produção de nanopartículas, como nanoemulsões, nanocápsulas e nanocompósitos (Martins et al., 2015). As nanoemulsões são formadas a partir de uma fração aquosa e uma lipídica e diferentes tipos de homogeneização (Assis et al., 2012). Já as nanocápsulas são produzidas para que a substância de interesse fique aprisionada em um núcleo protegido por uma parede estável (Ozkan et al., 2019). Os nanocompósitos são formados por materiais bifásicos, sendo que apenas uma das fases possui escala nanométrica (Masson \& Belino, 2021). As nanopartículas lipídicas sólidas, por sua vez, são coloides responsáveis pela proteção externa de componentes com propriedades funcionais (Gonçalves, 2018).

\subsection{Sensores}

O desenvolvimento de sensores baseia-se na aplicação de dispositivos de detecção em conjunto com transdutores, de forma a transmitir e amplificar sinais encontrados na interação de diferentes agentes, para posterior conversão, processamento e aplicação em diversos setores. Ao selecionar um sensor para um processo deve-se optar pela melhor seletividade, limite de detecção, estabilidade e sensibilidade (Sharma et al., 2021).

A preocupação com a contaminação química e adulteração dos alimentos faz com que questões de segurança alimentar sejam emergentes, gerando a busca pelo desenvolvimento de tecnologias para melhorar o processamento de alimentos. Dessa forma, o monitoramento dos produtos alimentícios auxilia na proteção dos consumidores contra possíveis riscos de adulteração, contaminação ou deterioração (Portorti et al., 2018; Luo et al., 2020).

Nanosensores podem ser agentes químicos ou mecânicos que apresentam elementos na escala nanométrica, construídos para indicar a presença de um determinado analito ou alguma característica ambiental. Quando aplicados na análise de alimentos podem auxiliar na qualidade e segurança alimentar, identificando íons, componentes orgânicos ou inorgânicos, biomoléculas e características do meio (Zhang et al., 2018; Wang et al., 2020; Yang \& Duncan, 2021).

O progresso nas tecnologias envolvendo nanomateriais simplifica a construção de sensores aplicados na segurança alimentar (Govindhan et al., 2014; Manikandan et al., 2018). Dessa forma, detectores físico-químicos podem ser empregados para análise de uma ampla variedade de contaminantes alimentares. Quando comparados com outros métodos, suas principais vantagens são a alta seletividade, sensibilidade e curto tempo de análise (Arduini et al., 2016; Lv et al., 2018; Kannan \& Guo, 2020).

Nanosensores ópticos utilizam sua capacidade de converter informações químicas de biomoléculas em sinais adequados para deteç̧ão, atuando principalmente com propriedades fluorescentes, colorimétricas e espectroscópicas. Podem ser empregados para detecção microbiológica de bactérias e demais moléculas (Pu et al., 2020).

A partir de partículas nanometálicas, podem ser construídos sensores utilizados na deteç̧ão de componentes químicos. Como exemplo pode-se citar o uso de nanopartículas de ouro na detecção de melamina, composto aromático aplicado em utensílios domésticos e tintas, em alimentos (Dobrucka, 2020).

Nanosensores eletroquímicos baseiam-se em reações entre os produtos químicos nanofabricados e o analito, para produzir ou consumir íons e elétrons que podem ser mensurados por voltagem, corrente ou impedância. Por apresentarem alta sensibilidade, compatibilidade com tecnologias nanofabricadas, baixo custo de manutenção e rapidez nas análises, podem ser utilizados para diversos tipos de detecção nos setores agrícola e de alimentos, como identificação de carbosulfan, Salmonella 
typhi ou metais pesados (Srivastava et al., 2018).

O nariz eletrônico e a língua eletrônica atuam de forma análoga a percepção sensorial humana, empregando sensores funcionalizados com materiais quimiossensíveis para detectar odores e sabores em alimentos, bebidas, produtos agroindustriais ou fármacos. Estes dispositivos são constituídos de transistores baseados em nanomateriais que convertem potenciais elétricos na forma de sinais biológicos (Son \& Park, 2018).

Visando o acompanhamento e a supervisão de diferentes processos, a utilização de sensores apresenta-se como uma alternativa não destrutiva voltada para a quantificação de agentes patógenos em alimentos. Com o aprimoramento das técnicas e tecnologias desse ramo, é possível promover uma produção de maior precisão, voltada para o monitoramento da ação biótica e abiótica, resultando em maior aproveitamento e qualidade dos produtos (Fahey et al., 2021).

\subsection{Doces}

Materiais compostos de alguns metais de transição como prata, ferro e dióxido de titânio, não metais como selênio e silicatos ou metais alcalino-terrosos como cálcio e magnésio podem ser encontrados em diversos alimentos. Óxido de magnésio, dióxido de silício e dióxido de titânio são agentes antiaglomerantes, além de atuarem como aditivos de cor em gomas, balas e coberturas de doces (Sahoo et al., 2021).

Entre os óxidos metálicos mais utilizados em produtos alimentícios comerciais estão as nanopartículas de dióxido de titânio, adicionadas como agente clareador e branqueador em indústrias de balas e gomas (King et al., 2018), para melhorar as propriedades de brilho e, quando incorporados a um polímero, podem ser aplicados em embalagens aumentando a vida útil dos produtos (Bratovcic, 2020).

Compostos nanoparticulados orgânicos também são empregados em produtos alimentícios. Para a proteção de chocolates e doces contra a umidade, aprimoramento da textura e preservação da cor e do sabor podem ser utilizados os nanolaminatos, que consistem em camadas de filmes alimentícios em escala nanométrica elaborados com base em polissacarídeos, proteínas e lipídios (Biswal et al., 2012; Bhagat et al., 2015).

Nanopartículas lipídicas estão presentes em emulsões de bebidas, como refrigerantes, sucos e bebidas lácteas. Também podem ser utilizadas para encapsular bioativos hidrofóbicos com propriedades colorantes, antimicrobianas, antioxidantes e de sabor, apresentando como vantagem de aumentar a estabilidade física dos produtos e possuir aparência transparente (Piorkowski \& Mcclements, 2014; Shin et al., 2015; Mcclements \& Xiao, 2017).

\subsection{Produtos Cárneos e Embalagens}

O setor nacional de produção de proteína de origem animal, bem como os demais setores do agronegócio brasileiro, tem apresentado um crescimento significativo ao longo das últimas décadas. Em função do aumento populacional de países em desenvolvimento, espera-se que a demanda por alimentos de origem animal cresça consideravelmente durante os próximos anos, uma realidade promissora para o setor de carnes mundial (Ferreira \& Filho, 2019). Segundo Mello (2017), a proteína de origem animal é o segundo maior produto de exportação do agronegócio nacional, perdendo apenas para a soja.

As exigências do mercado nacional e internacional relacionadas a qualidade de produtos cárneos estão cada vez maiores. Para a indústria alimentícia, a importância da qualidade dos produtos está diretamente relacionada com a satisfação por parte dos consumidores, além da saúde e segurança alimentar (Mello, 2017; Telles, 2014).

A conservação dos alimentos, com o intuito de impedir a contaminação por agentes patógenos e a deterioração dos mesmos, é uma das maiores preocupações das indústrias alimentícias. Dessa forma, a nanotecnologia apresenta-se como uma alternativa promissora para o aumento da vida de prateleira de alimentos, além de possuir uma série de aplicações com o propósito de trazer melhorias aos produtos e benefícios aos consumidores (Pinelli, 2018; Gonçalves, 2018). Algumas 
melhorias envolvem potenciais diminuições na utilização de sal, conservantes, gorduras e surfactantes em formulações alimentícias, assim como a elaboração ou aprimoramento de sabores e texturas (Chaudhry \& Castle, 2011).

A nanotecnologia aplicada a produtos cárneos tem sido voltada principalmente para o aumento do shelf life dos mesmos, por ser uma matriz altamente perecível e gerar grandes perdas para a indústria, além ser aplicada para promover o controle do crescimento microbiano nos mesmos. Pinelli (2018), em seus estudos, avaliou o uso de nanoemulssões de diferentes óleos essenciais para o controle de Clostridium sporogenes em mortadela, visando a aplicação das nanoemulsões como substitutos parciais de conservantes químicos nesses produtos embutidos, onde após a aplicação dos mesmos, não foram observadas alterações nas características físico-químicas do produto testado e em virtude da capacidade antimicrobiana dos óleos essenciais avaliados pela autora, as nanoemulsões mostraram-se promissoras para aplicação industrial.

Kazemeini et al. (2021), realizaram estudos aplicando a nanotecnologia em filés de peru, utilizando revestimentos comestíveis de alginato empregando nanoemulsões de óleo essencial, objetivando a inibição de Listeria monocytogenes.

Medeiros, et al. (2021) por meio de uma breve revisão da literatura, avaliaram o uso de nanopartículas de quitosana da conservação e produção de alimentos, como no uso em biofilmes ou bioembalagens comestíveis por conta da capacidade de formação de gel da quitosana e das suas propriedades antimicrobianas, que promovem a prolongação do prazo de validade de produtos, diminuindo o desperdício.

As embalagens também exercem um papel extremamente importante para a indústria de alimentos, sendo utilizados para conter os produtos e muito mais do que isso, promover a sua conservação, mantendo suas características inalteradas, sua qualidade e segurança para o consumidor, evitando a ação de microrganismos responsáveis pela deterioração do alimento, aumentando o shelf life do produto, impedindo também que fatores químicos e físicos degradem o mesmo (Jorge, 2013).

Um dos fatores mais importantes a serem observados em embalagens é a sua permeabilidade a gases, retenção de umidade e passagem de luz, pois, são fatores que influenciam diretamente na vida útil do alimento, principalmente cárneo. O oxigênio acelera o processo de rancidez da gordura presente no meio, proporcionando alterações organolépticas no produto, além de promover a oxidação de vitaminas, fazendo com que o valor nutricional do produto diminua consideravelmente, ocasionando também escurecimento por ação enzimática, além de promover a proliferação e ataque de patógenos que podem ser prejudiciais à saúde humana. Dessa forma, para proteger o alimento da ação do oxigênio no meio, utilizam-se embalagens que atuam como uma barreira para a passagem de gases, conservando o vácuo em seu interior (Mergen, 2004).

Levando esses fatores em consideração, Maurício (2020) em seus estudos, propôs a utilização alternativa para embalagens convencionais de filmes e revestimentos comestíveis contendo zeína e quitosana em sua formulação para a preservação de características desejáveis como a cor da carne, avaliada pelos consumidores no momento de compra do produto.

Para evitar a proliferação de patógenos em produtos alimentícios, principalmente os cárneos, nanopartículas com propriedades antimicrobianas podem ser incorporadas às embalagens, como é proposto por Arab et al. (2017), que em seus estudos, avaliaram a capacidade antimicrobiana de nanopartículas de óxido de zinco e óxido de cobre em Staphylococcus aureus e Salmonella, propondo a produção de embalagens com propriedades bactericidas.

Claro e Magalhães (2017), propuseram a síntese de nanopartículas de prata por seu alto poder antimicrobiano e a sua aplicação em filmes de nanocelulose por ser um polímero natural e bastante abundante, como embalagem alimentícia para a conservação do produto e preservação da qualidade do mesmo.

Dessa forma, percebe-se que o emprego de embalagens eficientes ajuda a impedir problemas relacionados a contaminações em produtos alimentícios, garantindo a qualidade e segurança alimentar. O uso da nanotecnologia embalagens pode oferecer soluções para problemas industriais, como o curto shelf life e possíveis ataques de patógenos (Brito et al. 2017; Jokar et al., 2012; Imran et al., 2010). 


\subsection{Frutas e Vegetais}

No âmbito das frutas e vegetais, a utilização da nanotecnologia também vem sendo bastante estudada. Além da aplicação em embalagens de alimentos como citado no item anterior, as alterações nas propriedades naturais das frutas e vegetais através da nanotecnologia podem tornar os seus nutrientes mais biodisponíveis, participar em nanoemulsões como sanitizantes, entre outros (Assis, 2011).

Sabe-se que a contaminação por microrganismos patogênicos, em especial de origem fúngica, é a responsável por cerca de $25 \%$ das perdas de frutas e demais vegetais pós-colheita (Sharma et al., 2009). Neste contexto, a utilização da nanotecnologia pode auxiliar na diminuição destas perdas.

A Tabela 1 apresenta uma relação de estudos que levaram em consideração a aplicação da nanotecnologia em frutas e vegetais. Através destes exemplos, denota-se a possibilidade de muitos estudos ainda serem realizados envolvendo a nanotecnologia e as frutas e vegetais.

Tabela 1. Estudos sobre a utilização da nanotecnologia em frutas e vegetais.

\begin{tabular}{|c|c|c|c|}
\hline Ação & Mecanismo & Resultado & Autor \\
\hline Aumento no shelf life de quiabos & $\begin{array}{l}\text { Nanoemulsão de alginato com óleo de } \\
\text { manjericão }\end{array}$ & $\begin{array}{l}\text { Eficaz no controle de } \\
\text { fungos deteriorantes }\end{array}$ & Gundewadi et al., 2018 \\
\hline $\begin{array}{l}\text { Aumento no shelf life de } \\
\text { morangos }\end{array}$ & $\begin{array}{l}\text { Nanopartículas de quitosana em gel de } \\
\text { proteção superficial de morangos }\end{array}$ & Maior efeito protetivo & Melo, 2020 \\
\hline $\begin{array}{c}\text { Comparação da ação } \\
\text { antimicrobiana de nanoemulsão e } \\
\text { extrato livre de erva-doce }\end{array}$ & Nanoemulsão de extrato de erva-doce & $\begin{array}{l}\text { Maior atividade } \\
\text { antimicrobiana }\end{array}$ & Ghazy et al., 2021 \\
\hline $\begin{array}{l}\text { Comparação do efeito } \\
\text { antioxidante de nanocápsulas de } \\
\text { óleo de menta }\end{array}$ & $\begin{array}{c}\text { Nanocápsulas de quitosana e óleo } \\
\text { essencial de menta }\end{array}$ & Maior efeito antioxidante & $\begin{array}{l}\text { Roshanpour et al., } \\
2021\end{array}$ \\
\hline Conservação de morangos & $\begin{array}{c}\text { Nanopartículas de prata com extrato de } \\
\text { alveloz }\end{array}$ & $\begin{array}{l}\text { Nanopartículas eficazes } \\
\text { contra a degradação } \\
\text { microbiológica }\end{array}$ & Oliveira, 2019 \\
\hline $\begin{array}{l}\text { Inibição de Listeria } \\
\text { monocytogenes }\end{array}$ & Nanoemulsão de óleo de alho e nisina & $\begin{array}{l}\text { Redução de } 4 \text { ciclos } \\
\text { logarítmicos de quatro das } \\
\text { cinco cepas analisadas }\end{array}$ & Pinilla, 2016 \\
\hline $\begin{array}{l}\text { Controle da podridão dos frutos } \\
\text { da pimenta malagueta causada } \\
\text { por Colletotrichum capsic }\end{array}$ & $\begin{array}{l}\text { Síntese de nanopartículas de cobre e } \\
\text { extratos de folhas de eucalipto e hortelã }\end{array}$ & $\begin{array}{l}\text { Inibição micelial de } \\
99,78 \%\end{array}$ & Iliger et al., 2021 \\
\hline
\end{tabular}

Fonte: Autores.

\subsection{Produtos Lácteos}

Nas indústrias lácteas as aplicações da nanotecnologia têm caminhado principalmente para o uso de embalagens ativas, tratamento de efluentes e controle de microrganismos. Os lácteos são produtos altamente nutritivos, disponíveis em inúmeras apresentações que podem ser utilizadas na produção de vários outros produtos. Devido a esta grande disponibilidade comercial para população, os laticínios tornam-se produtos de alta relevância para o mercado de alimentos. Entretanto, são produtos altamente perecíveis, principalmente quando disponíveis em produto fluido ou em apresentações com alta atividade de água, como queijos frescos e bebidas lácteas (Ayelén Vélez et al., 2017).

Erfanian et al. (2014), avaliaram a influência da redução nanométrica do cálcio, em leite pó, em relação a absorção e disponibilidade. Entre os resultados foi verificada a maior disponibilidade do cálcio com maior presença detectada nos tecidos ósseos do grupo alimentado com o produto nanotecnológico. Erfanian et al. (2017), compararam a biodisponibilidade do cálcio nanométrico em dois tipos diferentes de leites enriquecidos, constatando maior absorção do mineral em escala nano. Maurya e 
Aggarwal (2019), elaboraram uma bebida à base de leite com nanoestruturas encapsuladas de vitamina D3. Os autores constaram uma melhor aceitação sensorial do produto com vitamina encapsulada do que a amostra controle não encapsulada e não nanométrica.

O enriquecimento de produtos lácteos pelos sistemas de micro/nanoencapsulação pode fornecer propriedades funcionais a estes alimentos porque os óleos vegetais são fontes de ácidos graxos essenciais, como ácido docosahexaenóico, eicosapentaenóico e $\alpha$-linolênico (Delshadi et al., 2020). Iogurte, leite pasteurizado e queijo, podem ser enriquecidos por esta técnica. Também podem ser utilizados como antimicrobianos naturais em produtos alimentícios (Bedoya-Serna et al., 2018), conferindo propriedades funcionais a estes tipos de alimentos.

Outras utilidades estão sendo estudadas para aplicações em lácteos como a nanoemulsão de gordura em queijo cheddar (Sharma Khanal et al., 2019), queijo pasteurizado com aplicação de Óleo de chia (Salvia hispanica) (Cardoso et al., 2020), leite pasteurizado com aplicação do óleo de canela (Cinnamomum sp) (Bashiri et al., 2020).

\subsection{Legislação}

Até o presente, não há legislação brasileira regulamentadora sobre o uso da nanotecnologia no país. Tramita nas esferas legislativas um Projeto de Lei (880/2019), visando criar o Marco Legal da Nanotecnologia e Materiais Avançados (Brasil, 2021).

Algumas indústrias alimentícias baseiam-se em normativas já existentes de processos e produtos permitidos para assim utilizarem a nanotecnologia (Aguiar et al., 2021). Porém, faz-se necessário ampliar os estudos e a parceria entre o setor produtivo e os órgãos públicos para estabelecer normas que conciliem a segurança alimentar e a utilização da nanotecnologia em escala industrial (Barros, 2011).

\section{Considerações Finais}

Com tantas vantagens vistas na aplicação da nanotecnologia na área alimentícia, podemos concluir que a mesma possui grande eficiência quando bem aplicada. Dentre as vantagens de sua aplicação, o aumento da vida de prateleira dos produtos, embalagens inteligentes e a consequente redução do desperdício são fatores importantes para o atual mercado consumidor que vem buscando cada vez mais um consumo saudável e de menor agressividade ao meio ambiente.

Todavia, como entrave à maior utilização da nanotecnologia em alimentos, está a ausência de legislação regulamentadora destes produtos no Brasil, e uma possível toxicidade ao consumo humano. Dessa forma torna-se importante o investimento em pesquisas elucidativas a respeito dos efeitos de nano-produtos na engenharia de alimentos e o impacto dos mesmos na saúde e segurança alimentar.

\section{Referências}

Aguiar, J., Araújo, M., Durço, B. B., Portel, C. S., Pagani, M. M., Machado, M. T., Filho, E. R. T., \& Esmerino, E. A. (2021). Emprego da nanotecnologia como mecanismo de inovação tecnológica na indústria de alimentos: aplicações e desafios. Alimentos: Ciência, Tecnologia e Meio Ambiente. 1(12), 1-12.

Arab, F.E., Paris, E.C., de Souza, C. W. O., \& Ferreira, M. D. (2017). Avaliação do Efeito Antimicrobiano de Nanopartículas de Óxidos Metálicos. Embrapa Instrumentação, IX Workshop de Nanotecnologia Aplicada ao Agronegócio, 205-208.

Arduini, F., Cinti, S., Scognamiglio, V., \& Moscone, D. (2016). Nanomaterials in electrochemical biosensors for pesticide detection: advances and challenges in food analysis. Microchimica Acta, 183(07), 2063-2083.

Assis, O. B. G. (2011). Potenciais aplicações de nanotecnologia em alimentos. Embrapa Instrumentação-INFOTECA-E.

Ayelén Vélez, M., Cristina Perotti, M., Santiago, L., María Gennaro, A., \& Hynes, E. (2017). Bioactive compounds delivery using nano-technology: design and applications in dairy food. Nutrient Delivery, 221-250.

Azeredo, H. M. C. (2012). Fundamentos de Estabilidade de Alimentos. (2a ed.), Embrapa. 326. 
Barros, R. M. S. (2011). Nanoalimentos e nanotecnologias aplicadas a alimentos - riscos potenciais, necessidades regulatórias e proposta de instrumento para verificar opiniões sobre riscos potenciais à saúde e ao meio ambiente. Dissertação de Mestrado (Ciências na Saúde Pública). Escola Nacional de Saúde Pública Sergio Arouca, Rio de Janeiro.

Bashiri, S., Ghanbarzadeh, B., Ayaseh, A., Dehghannya, J., \& Ehsani, A. (2020). Preparation and characterization of chitosan-coated nanostructured lipid carriers (CH-NLC) containing cinnamon essential oil for enriching milk and antioxidant activity. Food Science and Technology, $119(11), 1-7$.

Bedoya-Serna, C. M., Dacanal, G. C., Fernandes, A. M., \& Pinho, S. C. (2018). Antifungal activity of nanoemulsions encapsulating oregano (Origanum vulgare) essential oil: in vitro study and application in Minas Padrão cheese. Brazilian Journal of Microbiology, 49(8), 929-935.

Bhagat, Y., Gangadhara, K., Rabinal, C., Chaudhari, G., \& Ugale, P. (2015). Nanotechnology in agriculture: a review. Journal of Pure and Applied Microbiology, 9, 737-747.

Biswal, S. K., Nayak, A. K., Parida, U. K., \& Nayak, P. L. (2012). Applications of nanotechnology in agriculture and food sciences. International Journal of Science Innovations and Discoveries, 2(1) 21-36.

Brasil, Senado Federal. Projeto de Lei $n^{o}$ 880/2019. https://www25.senado.leg.br/web/atividade/materias/-/materia/135353.

Bratovcic, A. (2020). Nanomaterials in Food Processing and Packaging, Its Toxicity and Food Labeling. Acta Scientific Nutritional Health, 4, 7-13.

Brito, S. da C., Sivieri, K., \& Ferreira, M. D. (2017). Avaliação da Atividade Antimicrobiana de Nanopartículas de Prata com Diferentes Concentrações em Filmes Poliméricos. Embrapa Instrumentação, IX Workshop de Nanotecnologia Aplicada ao Agronegócio, $173-176$.

Cardoso, L. G., Junior, I. J. B., Silva, R. V., Mossmann, J., Reinehr, C. O., Brião, V. B., \& Colla, L. M. (2020). Processed cheese with inulin and microencapsulated chia oil (Salvia hispanica). Food Bioscience, 37(1), 1-9.

Chaudhry, Q., \& Castle, L. (2011). Food Applications of Nanotechnologies: An Overview of Opportunities and Challenges for Developing Countries. Trends in Food Science \& Technology, 22, 595-603.

Claro, F. C., \& Magalhães, W. L. E. (2017). Síntese de Nanopartículas de Prata em Filmes de Nanocelulose. Embrapa Instrumentação, IX Workshop de Nanotecnologia Aplicada ao Agronegócio, 149-152.

Delshadi, R., Bahrami, A., Tafti, A. G., Barba, F. J., \& Williams, L L. (2020). Micro and nano-encapsulation of vegetable and essential oils to develop functional food products with improved nutritional profiles. Trends in Food Science \& Technology, 21(7), 1-27.

Dobrucka, R. (2020). Metal nanoparticles in nanosensors for food quality assurance. LogForum, 16(2) 271-278.

Erfanian, A., Rasti, B., \& Manap, Y. (2017). Comparing the calcium bioavailability from two types of nano-sized enriched milk using in-vivo assay. Food Chemistry, 214, 606-613.

Fahey, T., Pham, H., Gardi, A., Sabatini, R., Stefanelli, D., Goodwin, I., \& Lamb, D. W. (2021). Active and Passive Electro-Optical Sensors for Health Assessment in Food Crops. Sensors, 21(171), 1-39.

Fernandes, R. G., \& Oliveira, L. P. S. (2019). Entre riscos e desinformação: a utilização da nanotecnologia na indústria de alimentos. Revista Jurídica da FA7, $16(2), 63-81$.

Ferreira, M. D. P., \& Filho, J. E. R. V. (2019). Inserção no mercado nacional e a produção de carnes no Brasil. Instituto de Pesquisa Econômica Aplicada: Ipea, 43, Rio de Janeiro.

Ghazy, O. A., Fouad, M. T., Saleh, H. H., Kholif, A. E., \& Morsy, T. A. (2021). Ultrasound-assisted preparation of anise extract nanoemulsion and its bioactivity against different pathogenic bacteria. Food Chemistry, 341, 128259.

Gonçalves, R. A. dos S. Percepção do Consumidor em Relação à Nanotecnologia. (2018). 89. Dissertação (Mestrado em Ciências e Tec-nologia de Alimentos) - Programa de Pós-Graduação em Ciência e Tecnologia de Alimentos, Universidade Federal Rural do Rio de Janeiro.

Govindhan, M., Adhikari, B. R., \& Chen, A. (2014). Nanomaterials-based electrochemical detection of chemical contaminants. RSC Advances, 4(109), 6374163760 .

Greiner, R. (2009). Current and Projected Applications of Nanotechnology in the Food Sector. Nutrire, 34(1), $243-260$.

Gundewadi, G., Rudra, S. G., Sarkar, D. J., \& Singh, D. (2018). Nanoemulsion based alginate organic coating for shelflife extension of okra. Food Packaging and Shelf life, 18, 1-12.

Handford, C. E., Dean, M., Spence, M., Henchion, M., Elliott, C. T., \& Campbell, K. (2015). Awareness and Attitudes Towards the Emerging Use of Nanotechnology in the Agri-Food Sector. Food Control, 57 24-34.

Iliger, K. S., Sofi, T. A., Bhat, N. A., Ahanger, F. A., Sekhar, J. C., Elhendi, A. Z., Al-Huqail, A., \& Khan, F. (2021). Copper nanoparticles: Green synthesis and managing fruit rot disease of chilli caused by Colletotrichum capsici. Saudi Journal of Biological Sciences, $28(2), 1477-1486$.

Imran, M., Revol-Junelles, A. M., Martyn, A., Tehrany, E. A., Jacquot, M., Linder, M., \& Desobry, S. (2010). Active food packaging evolution: transformation from micro- to nanotechnology. Food Science \& Nutrition, 50(9), 799-821.

Jokar, M., Rahman, R. A., Ibrahim, N. A., Abdullah, L. C., \& Tan, C. P. (2012). Influence of nano-size reduction on absorption and bioavailability of calcium from melt production and antimicrobial efficiency of low-density polyethylene (ldpe)-silver nanocomposite film. Food and Bioprocess Technology, 5, 719728.

Jorge, N. (2013). Embalagens para alimentos. Cultura Acadêmica: Universidade Estadual Paulista, Pró-Reitoria de Graduação. 
Kannan, P., \& Guo, L. (2020). Nanosensors for food safety. In Nanosensors for Smart Cities, 339-354. Elsevier.

Kazemeini, H., Azizian, A., \& Adib, H. I. (2021). Inhibition of Listeria monocytogenes growth in turkey fillets by alginate edible coating with Trachyspermum ammi essential oil nano-emulsion. International Journal of Food Microbiology, 344, 109104.

King, T., Osmond-Mcleod, M. J., \& Duffy, L. L. (2018). Nanotechnology in the food sector and potential applications for the poultry industry. Trends in food science \& technology, 72, 62-73.

Luo, X., Han, Y., Chen, X., Tang, W., Yue, T., \& Li, Z. (2020). Carbon dots derived fluorescent nanosensors as versatile tools for food quality and safety assessment: A review. Trends in Food Science \& Technology, 95, 149-161.

Lv, M., Liu, Y., Geng, J., Kou, X., Xin, Z., \& Yang, D. (2018). Engineering nanomaterials-based biosensors for food safety detection. Biosensors and Bioelectronics, 106, 122-128.

Manikandan, V. S., Adhikari, B., \& Chen, A. (2018). Nanomaterial based electrochemical sensors for the safety and quality control of food and beverages, Analyst, 143(19), 4537-4554.

Martins, V. de C., de Oliveira Braga, E. C., de Oliveira Godoy, R. L., Borguini, R. G., Pacheco, S., de Araújo Santiago, M. C. P., \& de Ma-tos Nascimento, L. D. S. (2016). Nanotecnologia em alimentos: Uma breve revisão. Revista Eletrônica Perspectivas da Ciência e Tecnologia, 7(2), 25-42.

Masson, T. J., \& Belino, W. V. L. (2021). Estudo das propriedades termomecânicas de nanocompósitos híbridos. Brazilian Journal of Development, 7(4), $42618-42643$.

Maurício, R. A. Avaliação Sensorial de Carne Bovina e Ovina com Revestimento Comestível. (2020). 140. Dissertação (Mestrado em Alimentos e Nutrição) Programa de Pós-Graduação em Alimentos e Nutrição, Universidade Estadual Paulista Julio de Mesquita Filho.

Maurya, V. K., \& Aggarwal, M. (2019). Fabrication of nano-structured lipid carrier for encapsulation of vitamin D3 for fortification of 'Lassi'; A milk based beverage. Journal of Steroid Biochemistry and Molecular Biology, 193, 105429.

Mcclements, D. J., \& Xiao, H. (2017). Is nano safe in foods? Establishing the factors impacting the gastrointestinal fate and toxicity of organic and inorganic food-grade nanoparticles. npj Science of Food, 1(6), 1-13.

Medeiros, J. A. da C., Nunes, I. dos S., Araujo, A. S., Carvalho, E. A., \& Stamford, T. C. M. (2021). Nanopartículas de Quitosana na Conservação e Produção de Alimentos. Avanços em Ciência e Tecnologia de Alimentos, 4, 418-429.

Mello, F. R. (2017). Controle e Qualidade dos Alimentos. SAGAH.

Melo, N. F. C. B. Bioatividade de coberturas comestíveis a base de quitosana em gel e nanopartículas na conservação de morangos (Fra-garia x ananassa). (2020). 230. Tese (Doutorado em Nutrição) - Programa de Pós-graduação em Nutrição, Universidade Federal de Pernambuco.

Mergen, I. Z. Estudo da perda de vácuo em embalagens plásticas multicamadas para produtos cárneos curados cozidos. (2004). 132. Dissertação (Mestrado em Engenharia Química) - Programa de Pós-Graduação em Engenharia Química, Universidade Federal de Santa Catarina.

Moura, M. R., Mattoso, L. H. C., \& Zucolotto, V. (2012). Development of Cellulose-Based Bactericidal Nanocomposites containing Silver Nanoparticles and their Use as Active Food Packaging. Journal of Food Engineering, 109, 520-524.

Ozkan, G., Franco, P., Marco, I., Xiao, J., \& Capanoglu, E. (2019). A review of microencapsulation methods for food antioxidants: Principles, advantages, drawbacks and applications. Food Chemistry, 272(8), 494-506.

Pinelli, J. J. Uso de Nanoemulsões de Óleos Essenciais no Controle de Clostridium sporogenes em Mortadela. (2018). 115. Dissertação (Mestrado em Ciência dos Alimentos) - Programa de Pós-Graduação em Ciência dos Alimentos, Universidade Federal de Lavras.

Pinilla, C. M. B. Desenvolvimento, caracterização e avaliação da atividade antimicrobiana de nanolipossomas contendo nisina e extrato de alho (Allium sativum L.). (2016). 103. Dissertação (Mestrado em Microbiologia Agrícola e do Ambiente), Universidade Federal do Rio Grande do Sul.

Piorkowski, D. T., \& Mcclements, D. J. (2014). Beverage emulsions: recent developments in formulation, production, and applications. Food Hydrocolloids, $42,5-41$.

Potorti, A. G., Di Bella, G., Mottese, A. F., Bua, G. D., Fede, M. R., Sabatino, G., Salvo, A., Somma, R., Dugo, G., \& Lo Turco, V. (2018). Traceability of Protected Geographical Indication (PGI) Interdonato lemon pulps by chemometric analysis of the mineral composition. Journal of Food Composition and Analysis, 69, 122-128.

Pu, H., Xu, Y., Sun, D. W., Wei, Q., \& Li, X. (2020). Optical nanosensors for biofilm detection in the food industry: principles, applications and challenges. Critical Reviews in Food Science and Nutrition, 61(13), 2107-2124.

Roshanpour, S., Tavakoli, J., Beigmohammadi, F., \& Alaei, S. (2021). Improving Antioxidant Effect Of Phenolic Extract Of Mentha Piperita Using Nanoencapsulation Process. Journal Of Food Measurement And Characterization, 15(1), 23-32.

Sahoo, M., Vishwakarma, S., Panigrahi, C., \& Kumar, J. (2021). Nanotechnology: Current applications and future scope in food. Food Frontiers, 2 (1), 3-22.

Sharma, A., Ahmed, A., Singh, A., Oruganti, S., Khosla, A., \& Arya, S. (2021). Recent advances in tin oxide nanomaterials as electro-chemical/chemiresistive sensors. Journal of the Electrochemical Society, 168, 027505.

Sharma Khanal, B. K., Budiman, C., Hodson, M. P., Plan, M. R. R., Prakash, S., Bhandari, B., \& Bansal, N. (2019). Physico-chemical and biochemical properties of low-fat Cheddar cheese made from micron to nano sized milk fat emulsions. Journal of Food Engineering, 242, 94-105. 
Research, Society and Development, v. 11, n. 2, e3611225389, 2022

(CC BY 4.0) | ISSN 2525-3409 | DOI: http://dx.doi.org/10.33448/rsd-v11i2.25389

Sharma, R. R., Singh, D., \& Singh, R. (2009). Biological control of postharvest diseases of fruits and vegetables by microbial antagonists: A review. Biological control, 50(3), 205-221.

Shin, G. H., Kim, J. T., \& Park, H. J. (2015). Recent developments in nanoformulations of lipophilic functional foods. Trends Food Science and Technology, $46,144-157$.

Son, M., \& Park, T. H. (2018). The bioelectronic nose and tongue using olfactory and taste receptors: Analytical tools for food quality and safety assessment. Biotechnology advances, 36(2), 371-379.

Srivastava, A. K., Dev, A., \& Karmakar, S. (2018). Nanosensors and nanobiosensors in food and agriculture. Environmental Chemistry Letters, 16(1), 161182.

Telles, L. B. Ferramentas e sistemas de custo aplicados a gestão de qualidade no agronegócio. (2014). 68. Dissertação (Mestrado em Engenharia de Produção) - Programa de Pós-Graduação em Engenharia de Produção, Universidade Tecnológica Federal do Paraná, Ponta Grossa.

Wang, D., Wang, Z., Wang, X., Zhuang, X., Tian, C., Luan, F., \& Fu, X. (2020). Functionalized Copper Nanoclusters-Based Fluorescent Probe with Aggregation-Induced Emission Property for Selective Detection of Sulfide Ions in Food Additives. Journal of Agricultural and Food Chemistry, 68(40), 11301-11308.

Yang, T., \& Duncan, T. V. (2021). Challenges and potential solutions for nanosensors intended for use with foods. Nature nanotechnology, 16, $251-265$.

Zhang, L., Peng, D., Liang, R. P., \& Qiu, J. D. (2018). Graphene-based optical nanosensors for detection of heavy metal ions. Trends in Analytical Chemistry, $102,280-289$. 